\title{
Structural Distribution of Lennard-Jones Clusters Containing 562 to 1000 Atoms
}

\author{
Yuhong Xiang, Longjiu Cheng, Wensheng Cai, and Xueguang Shao* \\ Department of Chemistry, University of Science and Technology of China, Hefei, Anhui 230026, P. R. China
}

Received: May 21, 2004; In Final Form: August 13, 2004

\begin{abstract}
On the basis of the icosahedral and decahedral lattices, the lowest energies of the Lennard-Jones (LJ) clusters containing 562-1000 atoms with the two motifs are obtained by using a greedy search method (GSM). Energy comparison between the decahedra and icosahedra shows that icosahedral structures are predominant. However, most of the icosahedral structures with the central vacancy are more stable than that without the central vacancy. On the other hand, in the range of 562-1000 atoms, there are $41 \mathrm{LJ}$ clusters with the decahedral motif. The number of decahedra increases remarkably compared with the smaller LJ clusters. Consequently, the magic numbers and growth characters of decahedral clusters are also studied, and the results show that the magic numbers of intermediate decahedral clusters occur at $654,755,807,843,879,915$, and 935.
\end{abstract}

\section{Introduction}

Atomic clusters exhibit many surprising physical and chemical properties different from atom and bulk material because they are larger than ordinary atoms and smaller than ordinary bulk materials. The particularly complex and intriguing issues are the structures of the clusters and the dependence of structure on size. Because the electron diffraction data with finite size clusters obtained by using experimental methods do not fill all the space and do not possess translational symmetry, the analysis of structure relies to a large extent on comparisons between the measured diffraction patterns with the results of the theoretical calculations. Many experimental and theoretical investigations have been carried out on the structures of clusters. ${ }^{1-6}$

A primary objective of structural studies is the determination of size boundaries of energetic stability for various structural motifs and elucidation of the evolution process from the atom to condensed matter. The earlier investigations with electron diffraction data and analysis via molecular dynamics simulations employing Lennard-Jones (LJ) pair potential had established the presence of icosahedral structures for small LJ clusters containing 40-800 atoms and much bigger LJ clusters $\left(10^{4}-10^{5}\right.$ atoms) exhibit a crystalline face-centered-cubic structure. ${ }^{6}$ However, the optimal structures of LJ clusters in the intermediate regime have not been studied systematically.

On the other hand, the structural knowledge of intermediate size (containing from hundreds to thousands of atoms) clusters is important to achieve a deep understanding of clusters and reveal the evolution properties with cluster size. Therefore, it is interesting to study the stable structures of the intermediate size LJ clusters. The stable structures of the atomic clusters are the geometry with the lowest potential energy that is equivalent to the equilibrium structure at zero temperature. ${ }^{7}$ Therefore, finding the optimal structures of clusters is a global optimization problem of potential energy function.

In general, the global optimization methods used in cluster science can be generalized as two categories, unbiased and biased algorithms. Unbiased algorithms make no assumptions regarding cluster geometry and have been successfully applied

* Address correspondence to this author. Phone: +86-551-3606160. Fax: +86-551-3601592. E-mail: xshao@ustc.edu.cn. to LJ clusters, which are considered as a benchmark system for the optimization algorithm. For example, most of the global minima of LJ clusters up to $N=100$ ( $N$ is the cluster size) were found by an innovative GA approach that was proposed by Deaven and Ho (DH-GA). ${ }^{8}$ Hartke improved the DH-GA and found all the minima of LJ clusters up to 150 atoms. ${ }^{9}$ Basinhopping has been successfully applied to the optimization of LJ clusters up to $N=110,{ }^{10}$ and a variant of the basin-hopping method was applied for $N \leq 110$, which performed better than the original version. ${ }^{11}$ The parallel random tunneling algorithm (PRTA) was successfully applied to the optimization of LJ clusters and can find all the minima of LJ clusters with a size up to $200 .{ }^{12}$ All the known lowest energies up to $N=201$ were found by using the conformational space annealing (CSA) algorithm. ${ }^{13}$ However, there is still no unbiased global optimization method that can reach the intermediate size. This is due to the fact that the number of local minima tends to grow exponentially with cluster size $N .{ }^{14}$ The biased algorithms incorporated particular physical insights into the nature of cluster structure and improved largely the optimization efficiency. For example, Northby ${ }^{15}$ and Romero et al. ${ }^{16}$ have successfully located most of the optimal structures of LJ13-147 and LJ148-309 clusters, respectively. Moreover, in our previous work, ${ }^{17}$ the optimal structures of LJ310-561 clusters were successfully investigated.

The fcc structures are not competitive for intermediate size LJ clusters. ${ }^{17}$ Therefore, in this study, the fcc structures are not considered. On the basis of the lattice site coordinates of icosahedron and decahedron, the energy of each site was calculated by employing the Lennard-Jones potential. According to the energies, a greedy search method (GSM), i.e., iteratively to move the atom with the highest energy to the unoccupied site with the lowest energy, was developed and applied to the structural optimization of LJ562-1000 clusters. The putative lowest energies with decahedral and icosahedral motifs are obtained. Energy comparison between the two above motifs shows that icosahedral structures are predominant. However, icosahedra with the central vacancy ${ }^{18}$ (there is no atom at the center of the icosahedron) are more stable when the cluster size is larger than 752 with the only exception being LJ923. ${ }^{19}$ The number of decahedral structures in the studied LJ clusters 
increases remarkably compared with the smaller LJ clusters (there are only 14 clusters that are decahedral motifs when the size is smaller than 562). With the consideration of the importance of the decahedral structures in this size range, the magic numbers and growth characters of decahedral LJ clusters are also studied.

\section{Method}

In our previous work, the lattice construction method of Ino's decahedra and icosahedra was reported. ${ }^{17}$ In this study, the optimized cluster with $N$ atoms are distributed randomly on icosahedral or decahedral lattice including $N_{\mathrm{s}}$ lattice sites. By searching the appropriate sites for atoms to be occupied, the optimal structure with the lowest energy can be obtained. The growth of icosahedral and decahedral clusters is generally from inner to outer shells. Therefore, the lattice sites of icosahedral and decahedral cores, including $N_{\mathrm{c}}$ lattice sites, can be fixed in the optimization. The main procedures of the GSM based on lattice site energy can be summarized as follows:

(1) Generate an initial solution. Initially, the $N_{\mathrm{c}}$ atoms are placed on the lattice sites of a core, and then $N-N_{\mathrm{c}}$ atoms are distributed randomly on the remnant $N_{\mathrm{s}}-N_{\mathrm{c}}$ sites. This defines an initial configuration of the Lennard-Jones cluster, which can be represented by using a vector with $N_{\mathrm{s}}$ elements. Each element corresponds to a lattice site, whose value is 1 if the site is occupied by an atom, or 0 if it is not.

(2) Calculate the energy of each lattice site. According to the Cartesian coordinates of the lattice sites, the energy of the $i$ th lattice site can be calculated by employing Lennard-Jones potential

$$
E(i)=4 \epsilon \sum_{j \neq i}\left[\left(\frac{\sigma}{r_{i j}}\right)^{12}-\left(\frac{\sigma}{r_{i j}}\right)^{6}\right]
$$

where $r_{i j}, \epsilon$, and $2^{1 / 6} \sigma$ are the distance between lattice sites $i$ and $j$, the pair well depth, and the equilibrium pair separation, and $\epsilon=\sigma=1$ with reduced units are used.

(3) Move the atom with the highest energy to the unoccupied lattice site with the lowest energy. At first, the lattice sites with the lowest and the highest energy are found by energy comparison. Then, the value of the corresponding element of the occupied lattice site with the highest energy is set to 0 , and the value of the unoccupied site with the lowest energy is set to 1 . After the movement, the changes of lattice site energies are calculated and the energy of each lattice site updated.

(4) Calculate the total energy of the current configuration. The total energy of the cluster can be regarded as the energy sum of all the lattice sites occupied by atoms,

$$
E=\frac{1}{2} \sum_{i} E(i)
$$

therefore, the energy calculation is very fast because the $E(i)$ has already been calculated. If the total energy is decreased, the resolution will be taken as the starting configuration of the next iteration and the process will return to step 3. Otherwise, the process is terminated and the current solution is output as the best result.

The main procedure of GSM is iteratively moving the atom at the worst site to the best site. It may converge at various solutions. Therefore, to find the best solution, the GSM procedure will be repeated $N_{\text {try }}$ times from different random starting configurations. Because of the asymmetry of the outer atoms the force exerted onto the core is not symmetric, and the

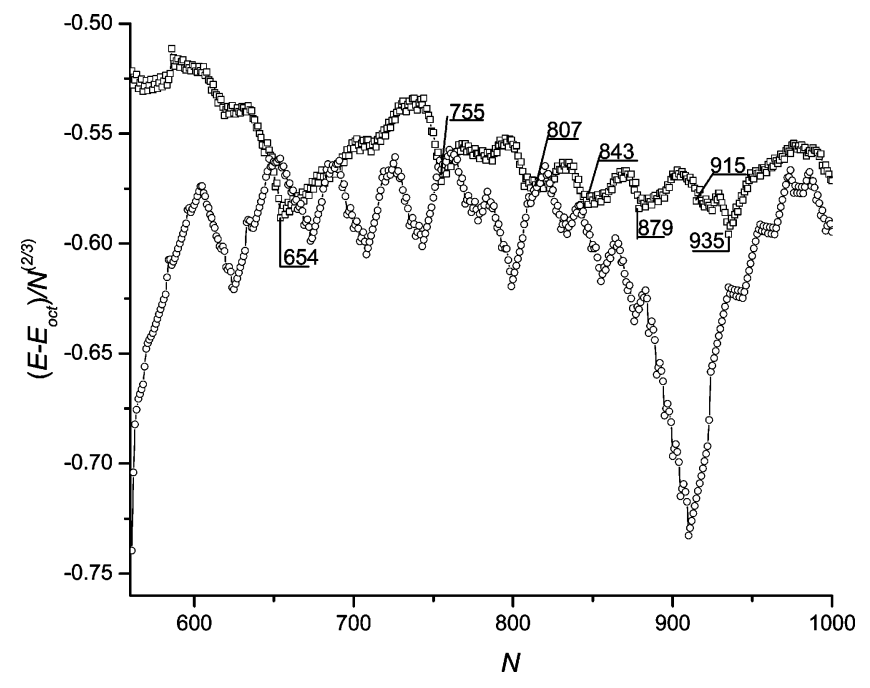

Figure 1. The energy comparison of complete sequence of icosahedra and decahedra. Marks' decahedron and icosahedron are plotted with $\square$ and $\bigcirc$, respectively. $N$ is the number of atoms, $E$ is the energy of the cluster with icosahedron and Marks' decahedron, and $E_{\text {oct }}$ is the fitting energy of the cuboctahedra.

positions of atoms may deviate slightly from the lattice sites. The local minimization technique, a limited memory BFGS (L-BFGS), ${ }^{20}$ is indispensable. Furthermore, the solution with the lowest energy is not always that with the lowest energy after the BFGS, therefore $N_{\text {best }}$ solutions with lower energies are recorded and local minimizations are performed to obtain the optimal structures of the LJ clusters. The solution with the lowest potential energy after BFGS will be selected as the final optimization result.

The GSM method is validated by optimization of LJ13-561 clusters with icosahedral and decahedral motifs based on icosahedral and Ino's decahedral lattices. Results show that the proposed method successfully located all the known lowest minima. Therefore, the optimization method proposed is reliable. On the other hand, the optimization time is shorten significantly because the energy calculation is simplified as a summation operation and the number of the time-consuming BFGS is only $N_{\text {best }}$ for a given size cluster. For example, on the basis of icosahedra with 922 lattice sites, the mean time consumed for successful optimization of LJ751 clusters is about $60 \mathrm{~min}$ (PIII $1 \mathrm{GHz})$.

\section{Results and Discussion}

3.1. The Optimization Results of LJ562-1000 Clusters. According to the reported works, icosahedral clusters are favored for the smallest cluster sizes and Marks' decahedra are favored for intermediate sizes, ${ }^{6}$ furthermore, most of the icosahedra with the central vacancy are more stable than those without the central vacancy in the range of 562-922 atoms. Therefore, all the LJ562-1000 clusters with decahedral and icosahedral motifs, with and without the central vacancy, are optimized with the proposed method. The energies of the most stable icosahedra are compared with that of decahedra. The comparison is given in Figure 1. Energies of clusters are plotted as $\left(E-E_{\text {oct }}\right) / N^{2 / 3}$ versus $N$, where $E$ is the energy of Marks' decahedron and icosahedron and $E_{\text {oct }}$ is the four-term least-squares fit to the binding energies of face centered cubic (fcc) cuboctahedra. ${ }^{21}$ From Figure 1, it can be seen that in the four ranges near 657, 686,758 , and 819 the decahedral energies of clusters are lower than those of icosahedra, whose decahedral energies are given in Table 1. 
TABLE 1: The Lowest Energies of LJ Clusters with the Decahedral Motif

\begin{tabular}{cccccccc}
\hline$N$ & energy/ $\epsilon$ & $N$ & energy/ $\epsilon$ & $N$ & Energy/ & $N$ & energy/ $\epsilon$ \\
\hline 650 & -4490.882106 & 682 & -4729.812239 & 754 & -5267.902856 & 815 & -5727.254474 \\
651 & -4498.828911 & 683 & -4737.032869 & 755 & -5276.024042 & 816 & -5734.477393 \\
652 & -4506.152061 & 684 & -4744.376499 & 756 & -5283.248304 & 818 & -5749.670074 \\
653 & -4514.127901 & 685 & -4752.053386 & 757 & -5290.488239 & 819 & -5757.354578 \\
654 & -4522.237084 & 686 & -4759.274013 & 758 & -5298.253262 & 820 & -5764.577273 \\
655 & -4529.460139 & 687 & -4766.617576 & 759 & -5305.477507 & 821 & -5772.084288 \\
656 & -4536.712799 & 688 & -4774.294327 & 760 & -5312.718230 & 822 & -5779.768096 \\
657 & -4544.483116 & 689 & -4781.514121 & 761 & -5320.483317 & 823 & -5787.452061 \\
658 & -4551.706161 & 691 & -4796.534940 & 762 & -5327.706748 & &
\end{tabular}

$659-4558.959717$

$660-4566.730001$

$661 \quad-4573.952330$

$662-4581.204895$

$663-4588.975484$

$664 \quad-4596.197078$

Determination of size boundaries of energetic stability for various structural motifs is a primary objective of structural studies, and in the past, the crossover size was obtained by extrapolating the lowest energy sequence of different motifs. $6,21,22$ However, from Figure 1, it is clear that the lowest energy sequence of icosahedra severely overestimates the icosahedral stability of the LJ clusters compared with those of decahedra. Moreover, the complete energy sequence of Marks' decahedra in the range of 562-1000 atoms fluctuates more smoothly relative to that of icosahedra, especially when the sizes near their magic numbers. Therefore, it is not rigorous enough to extrapolate the crossover size with use of the lowest energy sequence.

3.2. Structural Characters of LJ562-1000 Clusters. The optimization results of LJ562-1000 clusters show that the icosahedral structures are predominant. However, it is noteworthy that the structures with the central vacancy are stable in most of the icosahedral LJ562-1000 clusters because of the effect of icosahedral strain, ${ }^{23}$ which can be obtained by comparing the energies of icosahedral LJ clusters, with and without the central vacancy, with the same atom number. The results show that all the icosahedral structures have the central vacancy when the cluster size is larger than 752 with the only exception being LJ923. This is because if the atom is located on the outer layer of the icosahedral LJ922 cluster with the central vacancy, fewer nearest neighbors make it very unstable. Moreover, the LJ923 cluster without the central vacancy is not stable because of the icosahedral strain. It can be seen from the first finite difference of energy, as shown in Figure 2, which

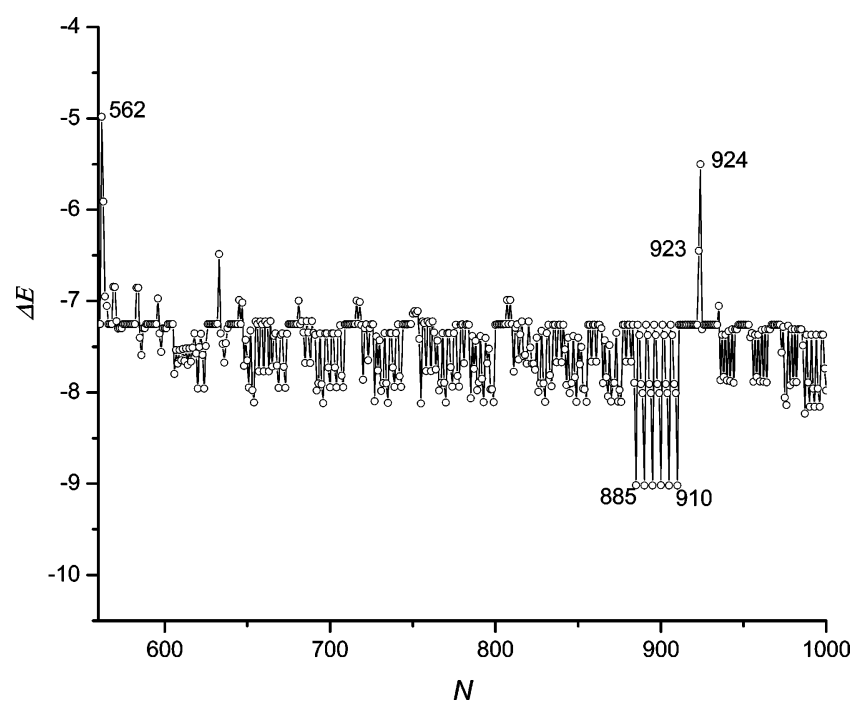

Figure 2. Plot of the variation of the first finite differences of energy, $\Delta E=E(N)-E(N-1)$, versus cluster size $N$. indicates the relative stability of the cluster compared with that its neighbors. ${ }^{12}$ On the other hand, from Figure 2, it is clear that the structures of LJ885, LJ890, LJ895, LJ900, LJ905, and LJ910 are very stable, which corresponds to icosahedron without the vertex atoms and icosahedra without the five atoms around the vertex of the outermost shell.

Although the formation of the central vacancy effectively relieves the effect of the strain of the icosahedral configuration, the number of configurations with the decahedral motif, which is characterized by a smaller strain energy, increases remarkably. Moreover, it is worth noting that the optimal structures of LJ656 and LJ686 are not the pentakaitetrakontahedra (PTK) and the complete Marks' decahedral configurations. ${ }^{6,22,24}$ The structural differences are illustrated in Figure 3. The energy difference is $0.029618 \epsilon$ and $0.538432 \epsilon$, respectively. Therefore, in the case of the structural study of clusters, the optimization procedure based on lattice construction is necessary.

The structural distribution of LJ clusters is given in Table 2. It clearly points out the structure of each cluster in the range of 562-1000 atoms.
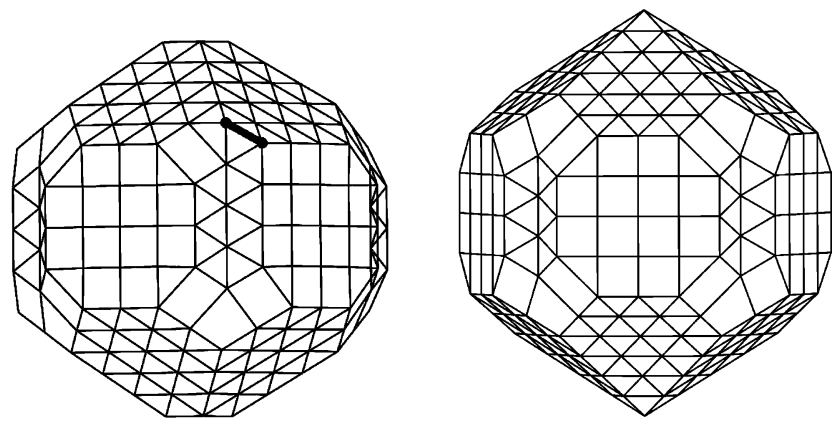

LJ656
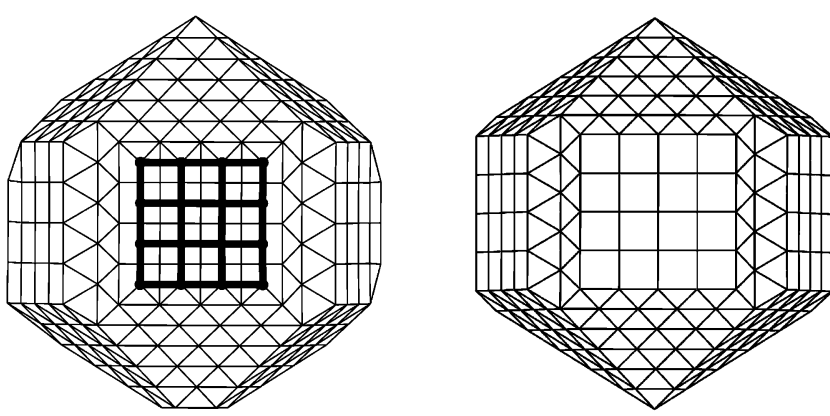

LJ686

Figure 3. The illustrations of the optimal structures of LJ656 and LJ686 obtained in this study (left), and the PTK and complete Marks' decahedron obtained by using the modeling method (right). 
TABLE 2: Structural Distribution of LJ562-1000 Clusters

\begin{tabular}{lccc}
\hline & & \multicolumn{2}{c}{$N$} \\
\cline { 3 - 4 } \multicolumn{1}{c}{ structure } & $n^{\mathrm{a}}$ & without the central vacancy & with the central vacancy \\
\hline icosahedron & 398 & $562-584,586-596,598-605,607,609,611,613$, & $585,597,606,608,610,612,614,616,619-620$, \\
& & $615,617-618,621-622,625-633,635,637-647$, & $623-624,634,636,648,665,668-669,672-673$, \\
& & $649,666-667,670-671,674-681,706,709-713$, & $690,692-705,707-708,714,716,718-749,751$, \\
& & $715,717,750,752,923$. & $753,763-814,817,824-922,924-1000$. \\
Marks' decahedron & 41 & $650-664,682-689,691,754-762,815-816,818-823$. &
\end{tabular}

${ }^{a}$ The total number of LJ clusters with icosahedral or decahedral structures.

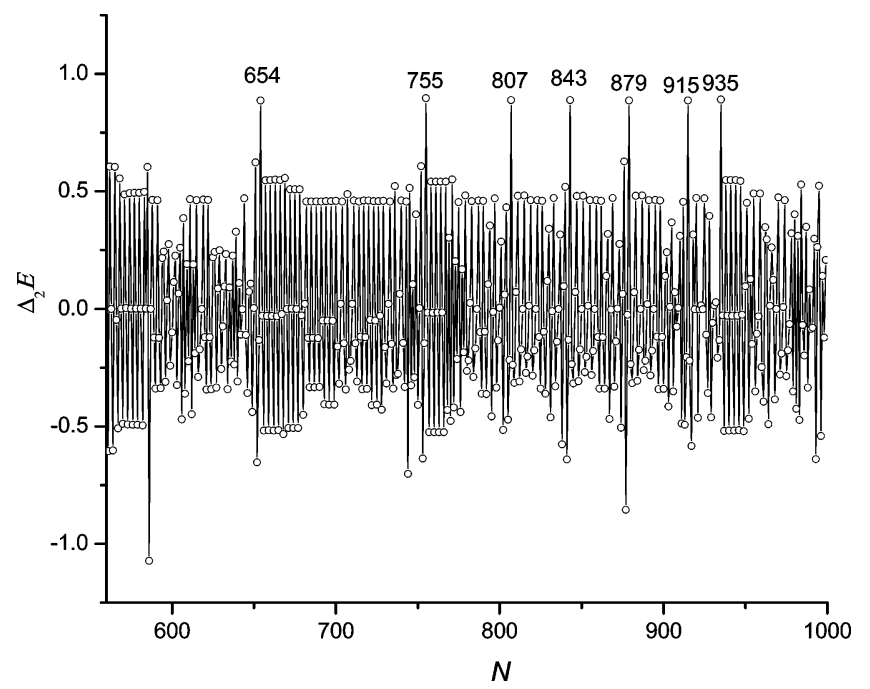

Figure 4. Plot of the second finite energy differences with decahedral motifs, $\Delta_{2} E(N)=E(N+1)+E(N-1)-2 E(N)$, versus the size of cluster $N$. The peaks correspond to the particularly stable structures.

3.3. Magic Numbers and Growth Characters of Decahedral LJ Clusters. From the optimization results of LJ clusters, it is clear that the number of decahedra in the range of $562-1000$ atoms increases remarkably. Consequently, it is instructive to comprehend the decahedral structure characters. To investigate the magic numbers of decahedral LJ clusters, the second finite differences of energy, ${ }^{25,26} \Delta_{2} E(N)=E(N+$ 1) $+E(N-1)-2 E(N)$, were calculated and are shown in Figure 4, where $E(N)$ is the energetic minimum of the cluster with $N$ atoms. The positive peaks in Figure 4 correspond to the clusters that are particularly stable compare to adjacent sizes. From Figure 4, it can be found that remarkable positive peaks occur at $N=654,755,807,843,879,915,935$ which are consistent with the local minima of the energy curve of decahedra as shown in Figure 1. All these structures have no atoms on twinning edges between capping (111) faces and reentrant (111) faces and on the vertex site. These magic numbers should be useful in studying other decahedral clusters.

The decahedral growth characters can be obtained by careful investigation of the structural transformation. For example, the growth process from LJ654 to LJ755 can be described as follows. Initially, additional atoms locate on twinning edges between capping (111) faces and reentrant (111) faces and the vertex site. Then, when the additional atom number reaches a critical size, the atoms occupy the outer (100) faces one by one, which has been noted in the research of magic numbers in small decahedral clusters. ${ }^{27}$ Finally, all the atoms cap on the capping (111) faces. Therefore, the configuration changes from approximately spherical to oblate to prolate structures. To obtain the stable spherical structures, the grow sequence from LJ755 to LJ771, LJ807, LJ843, LJ879 and to LJ935 also processes by the capping of the (100) face one by one, which can be clearly illustrated by the projections of cluster structures in Figure 5.
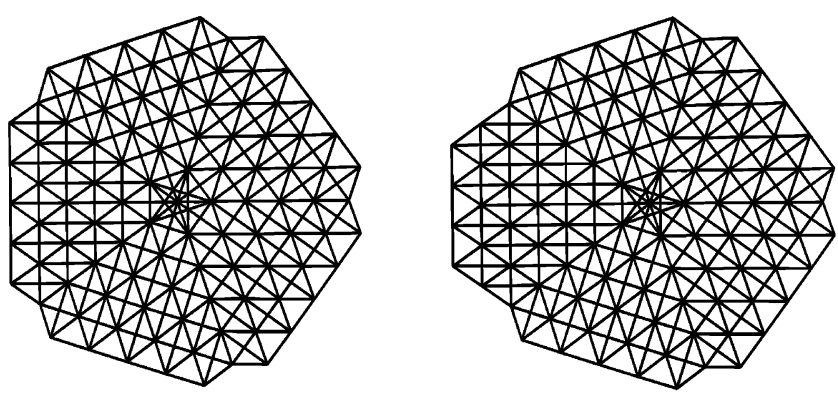

LJ755

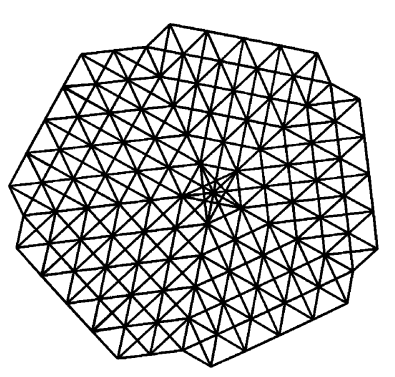

LJ771

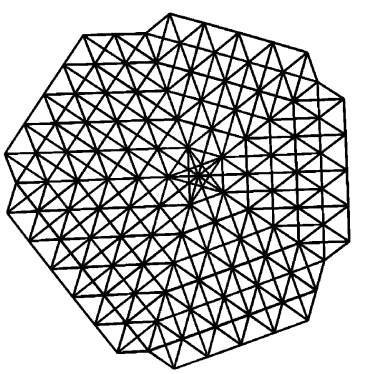

LJ807

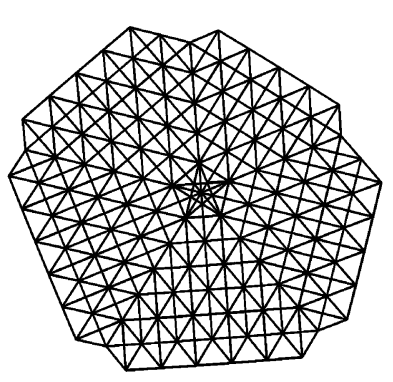

LJ879

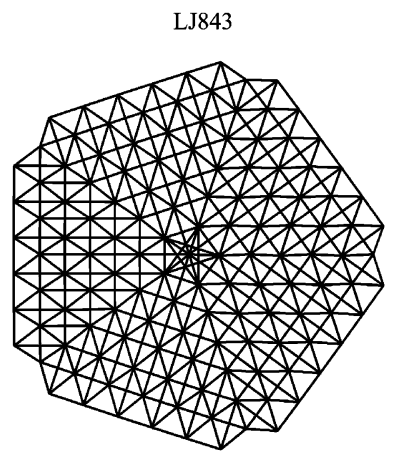

LJ935

Figure 5. The schematics of growth process with use of the plane projections of decahedral LJ clusters.

All these growth characters provide a good starting point for understanding the decahedral structures of intermediate size clusters.

\section{Conclusions}

By using the greedy search method based on lattice site energy, the lowest energies of LJ562-1000 clusters with decahedral and icosahedral motifs are obtained. By comparing the energies of the LJ cluster with those of the same size, it is found that icosahedral structures are predominant in this range. However, icosahedral structures with the central vacancy are favorable in most of the LJ562-1000 clusters because of the existence of icosahedral strain. Although the formation of the central vacancy relieves the effect of icosahedral strain, 41 clusters with decahedral motif are more stable than the same size icosahedra. On the other hand, because of the competition 
of decahedra in the size of LJ clusters studied, the magic numbers and growth characters of decahedra are investigated by analyzing the second finite differences of optimal decahedral structures. The structural knowledge of intermediate size clusters is helpful in achieving a deep understanding of clusters and revealing the evolution properties with cluster size.

Acknowledgment. This study is supported by the outstanding youth fund (No. 20325517) from the National Natural Scientific Foundation of China (NNSFC) and the Teaching and Research Award Program for Outstanding Young Teachers (TRAPOYT) in higher education institutions of the Ministry of Education (MOE), P. R. China.

Supporting Information Available: Listing of the energies of the putative global minima of LJ562-1000 clusters. This material is available free of charge via the Internet at http:// pubs.acs.org.

\section{References and Notes} 3458.

(1) Farges, J.; Raoult, B.; Torchet, G. J. Chem. Phys. 1973, 59, 3454-

(2) Farges, J.; De Feraudy, M. F.; Raoult, B.; Torchet, G. J. Chem. Phys. 1983, 78, 5067-5080.

(3) Farges, J.; De Feraudy, M. F.; Raoult, B.; Torchet, G. J. Chem. Phys. 1986, 84, 3491-3501.

(4) Van de Waal, B. W.; Torchet, G.; De Feraudy, M. F. Chem. Phys. Lett. 2000, 331, 57-63.

(5) Marks, L. D. Philos. Mag. A 1984, 49, 81-93.

(6) Raoult, B.; Farges, J.; De Feraudy, M. F.; Torchet, G. Philos. Mag. B 1989, 60, 881-906.
(7) Doye, J. P. K.; Calvo, F. J. Chem. Phys. 2002, 116, 8307-8317.

(8) Deaven, D. M.; Tit, N.; Morris, J. R.; Ho, K. M. Chem. Phys. Lett. 1996, 256, 195-200

(9) Hartke, B. J. Comput. Chem. 1999, 20, 1752-1759.

(10) Wales, D. J.; Doye, J. P. K. J. Phys. Chem. A 1997, 101, 51115116.

(11) Leary, R. H. J. Global Optim. 2000, 18, 367-383.

(12) Shao, X. G.; Jiang, H. Y.; Cai, W. S. J. Chem. Inf. Comput. Sci. 2004, 44, 193-199.

(13) Lee, J.; Lee, I. H.; Lee, J. Phys. Rev. Lett. 2003, 91, 080201080204.

(14) Wales, D. J.; Scheraga, H. A. Science 1999, 285, 1368-1372.

(15) Northby, J. A. J. Chem. Phys. 1987, 87, 6166-6177.

(16) Romero, D.; Barron, C.; Gomez, S. Comput. Phys. Commun. 1999, $123,87-96$

(17) Xiang, Y. H.; Jiang, H. Y.; Cai, W. S.; Shao, X. G. J Phys. Chem. A 2004, 108, 3586-3592.

(18) Boyer, L. L.; Broughton, J. Q. Phys. Rev. B 1990, 42 1146111468

(19) Shao, X. G.; Xiang, Y. H.; Cai, W. S. Chem. Phys. 2004, 305, 69-75.

(20) Liu, D. C.; Nocedal, J. Math. Progm. 1989, 45, 503-528.

(21) Xie, J.; Northby, J. A.; Freeman, D. L.; Doll, J. D. J. Chem. Phys. 1989, 91, 612-619.

(22) Cleveland, C. L.; Landman, U. J. Chem. Phys. 1991, 94, 73767396.

(23) Farges, J.; De Feraudy, M. F.; Raoult, B.; Torchet, G. Acta Crystallogr. A 1982, 38, 656-663.

(24) Jiang, H. Y.; Cai, W. S.; Shao, X. G. J. Phys. Chem. A 2003, 107, $4238-4243$.

(25) Cai, W. S.; Feng, Y.; Shao, X. G.; Pan, Z. X. Chem. Phys. Lett. 2002, 359, 27-34.

(26) Doye, J. P. K.; Wales, D. J. Chem. Phys. Lett. 1996, 262, 167174

(27) Doye, J. P. K.; Wales, D. J. Chem. Phys. Lett. 1995, 247, 339- 